\title{
Global existence and blow-up to the solutions of a singular porous medium equation with critical initial energy
}

Lirong Luo and Jun Zhou*

"Correspondence:

jzhouwm@163.com

School of Mathematics and

Statistics, Southwest University,

Chongqing, 400715, P.R. China

\begin{abstract}
This paper is devoted to the study of a singular porous medium equation, which was studied extensively in recent years. We obtain the global existence and blow-up condition at the critical initial energy $E\left(u_{0}\right)=d$, while the previous papers only considered the case $E\left(u_{0}\right)<d$, where $d$ is a positive constant which will be given in the main part of this paper.
\end{abstract}

MSC: 35B33; 35K50; 35K55; 35K63

Keywords: singular porous medium equation; critical initial energy; global existence; blow-up

\section{Introduction}

Suppose a compressible fluid flows in a homogeneous isotropic rigid porous medium. Then the volumetric moisture content $\theta(x)$, the macroscopic velocity $\vec{V}$ and the density of the fluid $\rho$ are governed by the following equation $[1,2]$ :

$$
\theta(x) \frac{\partial \rho}{\partial t}+\operatorname{div}(\rho \vec{V})-f(\rho)=0
$$

where $f(u)$ is the source. From Darcy's law, one has the following relation:

$$
\rho \vec{V}=-\lambda \nabla P,
$$

where $\rho \vec{V}$ and $P$ denote the momentum velocity and pressure, respectively, $\lambda>0$ is some physical constant.

If the fluid considered is the polytropic gas, then the pressure and density satisfy the following equation of the state:

$$
P=c \rho^{\gamma}
$$

where $c>0, \gamma>0$ are some constants. Thus, it follows from (1.1)-(1.3) that

$$
\theta(x) \frac{\partial \rho}{\partial t}=c \lambda \Delta\left(\rho^{\gamma}\right)+f(\rho) .
$$

(c) 2016 Luo and Zhou. This article is distributed under the terms of the Creative Commons Attribution 4.0 International License (http://creativecommons.org/licenses/by/4.0/), which permits unrestricted use, distribution, and reproduction in any medium, provided you give appropriate credit to the original author(s) and the source, provide a link to the Creative Commons license, and indicate if changes were made. 
In this paper, we consider (1.4) with $\theta(x)=|x|^{-\delta}$ and $f(\rho)=\rho^{\sigma}$. Furthermore, we incorporate zero boundary condition to this problem. Then we get the following initial-boundary problem after changing variables and notations:

$$
\left\{\begin{array}{l}
|x|^{-s} \frac{\partial u}{\partial t}-\Delta u^{m}=u^{p-1}, \quad(x, t) \in \Omega \times(0, T) \\
u(x, t)=0, \quad(x, t) \in \partial \Omega \times(0, T) \\
u(x, 0)=u_{0}(x), \quad x \in \Omega
\end{array}\right.
$$

where $u_{0} \in H_{0}^{1}(\Omega)$ is a nonnegative and nontrivial function, $T \in(0, \infty], \Omega$ is a bounded domain in $\mathbb{R}^{N}(N \geq 3)$ with smooth boundary $\partial \Omega, m \geq 1,0 \leq s \leq 1+1 / m \leq 2, m<p-1 \leq$ $\frac{(N+2) m}{N-2}$.

Problem (1.5) and the related models were studied in [2-8], in order to introduce the main results of [5], we need the following functionals and sets, which were given in [5].

- A function $u$ is called a solution of (1.5) if

$$
u^{m} \in L^{\infty}\left(0, T ; H_{0}^{1}(\Omega)\right), \quad \int_{0}^{T}\left\||x|^{-\frac{s}{2}}\left(u^{\frac{m+1}{2}}\right)_{t}\right\|_{2}^{2} d t<+\infty,
$$

and $u$ satisfies (1.5) in the distribution sense.

- The energy functional related to the stationary equation

$$
E(u)=\frac{1}{2 m} \int_{\Omega}\left|\nabla u^{m}\right|^{2} d x-\frac{1}{m+p-1} \int_{\Omega}|u|^{m+p-1} d x, \quad u^{m} \in H_{0}^{1}(\Omega) .
$$

- The Nehari functional

$$
H(u)=\int_{\Omega}\left|\nabla u^{m}\right|^{2} d x-\int_{\Omega}|u|^{m+p-1} d x, \quad u^{m} \in H_{0}^{1}(\Omega) .
$$

- The Nehari manifold

$$
K=\left\{u: u^{m} \in H_{0}^{1}(\Omega), H(u)=0, u \neq 0\right\} .
$$

- The potential depth

$$
\begin{aligned}
d & =\inf \left\{\sup _{\lambda \geq 0} E(\lambda u): u^{m} \in H_{0}^{1}(\Omega), u \neq 0\right\} \\
& =\inf _{u \in K} E(u)=\frac{p-1-m}{2 m(m+p-1)} C^{\frac{-2(m+p-1)}{p-1-m}},
\end{aligned}
$$

where $C$ is the optimal constant of the Sobolev embedding $H_{0}^{1}(\Omega) \subset L^{\frac{m+p-1}{m}}(\Omega)$. Particularly we have

$$
\left\|u^{m}\right\|_{\frac{m+p-1}{m}} \leq C\left\|\nabla u^{m}\right\|_{2}
$$

for $u^{m} \in H_{0}^{1}(\Omega)$ since $m<p-1 \leq \frac{(N+2) m}{N-2}$, where $\|\cdot\|_{r}$ denotes the norm of $L^{r}(\Omega)$. 
- The sets related to global existence and blow-up

$$
\begin{aligned}
& \Sigma_{1}=\left\{u: u^{m} \in H_{0}^{1}(\Omega), E(u)<d, H(u)>0\right\} \cup\{0\}, \\
& \Sigma_{2}=\left\{u: u^{m} \in H_{0}^{1}(\Omega), E(u)<d, H(u)<0\right\} .
\end{aligned}
$$

The solution $u(x, t)$ of problem (1.5) is called blow-up at finite time $T$ if $\|u\|_{L^{\infty}(\Omega)} \rightarrow+\infty$ as $t \rightarrow T_{-}$. Otherwise, we say $u(x, t)$ exists globally. The following are the main results of [5].

Theorem 1.1 If $u_{0} \in \Sigma_{1}$, then the solution $u$ to the problem (1.5) exists globally; if $u_{0} \in \Sigma_{2}$, then $u$ blows up at finite time.

In view of the above results, we may ask if the solution of $u$ of the problem (1.5) blows up or exists globally when $E\left(u_{0}\right) \geq d$. The main task of this paper is to answer the question for $E\left(u_{0}\right)=d$. In order to give the main results of the present paper, we introduce two sets as follows:

$$
\begin{aligned}
& \mathcal{S}=\left\{u: u^{m} \in H_{0}^{1}(\Omega),\left\|\nabla u^{m}\right\|_{2}<\left(\frac{2 m(m+p-1)}{p-1-m} d\right)^{\frac{1}{2}}\right\}, \\
& \mathcal{B}=\left\{u: u^{m} \in H_{0}^{1}(\Omega),\left\|\nabla u^{m}\right\|_{2}>\left(\frac{2 m(m+p-1)}{p-1-m} d\right)^{\frac{1}{2}}\right\} .
\end{aligned}
$$

Then

$$
\partial \mathcal{S}=\partial \mathcal{B}=\left\{u: u^{m} \in H_{0}^{1}(\Omega),\left\|\nabla u^{m}\right\|_{2}=\left(\frac{2 m(m+p-1)}{p-1-m} d\right)^{\frac{1}{2}}\right\} .
$$

The main results of this paper are the following theorem.

Theorem 1.2 Assume $E\left(u_{0}\right)=d$, then we have

1. if $u_{0} \in \mathcal{S}$, then the problem (1.5) admits a global solution $u$ such that $u^{m}(t) \in L^{\infty}\left(0,+\infty ; H_{0}^{1}(\Omega)\right)$ and $u(t) \in \overline{\mathcal{S}}=\mathcal{S} \cup \partial \mathcal{S}$ for $0 \leq t<+\infty$;

2. if $u_{0} \in \mathcal{B}$, then the solution of problem (1.5) will blow up at finite time.

\section{Proof of Theorem 1.2}

In this section, we will prove Theorem 1.2. First of all, we will introduce some useful lemmas.

Lemma 2.1 Assume the function $u \not \equiv 0$ satisfying $u^{m} \in H_{0}^{1}(\Omega)$. Then there exists a unique positive value $\mu_{*}$ defined as

$$
\mu_{*}=\sqrt[p-m-1]{\frac{\int_{\Omega}\left|\nabla u^{m}\right|^{2} d x}{\int_{\Omega}|u|^{m+p-1} d x}}
$$

such that $E(\mu u)$ is strictly increasing for $0<\mu<\mu_{*}$, strictly decreasing for $\mu_{*}<\mu<\infty$.

Proof From

$$
E(\mu u)=\mu^{2 m}\left[\frac{1}{2 m}\left\|\nabla u^{m}\right\|_{2}^{2}-\frac{\mu^{p-m-1}}{m+p-1}\|u\|_{m+p-1}^{m+p-1}\right]
$$


and $p>m+1$ we get $\lim _{\mu \rightarrow 0} E(\mu u)=0, \lim _{\mu \rightarrow+\infty} E(\mu u)=-\infty$. Furthermore, since $\mu=\mu_{*}$ is the unique positive root of the equation $\frac{d E(\mu u)}{d \mu}=0$, the conclusion follows.

Lemma 2.2 Let $\mathcal{S}, \mathcal{B}, \partial \mathcal{S}$, and $\partial \mathcal{B}$ be the sets defined as (1.12) and (1.13).

(i) If $u \in \mathcal{S}$ and $\left\|\nabla u^{m}\right\|_{2} \neq 0$, then $\left\|\nabla u^{m}\right\|_{2}^{2}>\left\|u^{m}\right\|_{\frac{m+p-1}{m}}^{\frac{m+p-1}{m}}$.

(ii) If $u \in \partial \mathcal{S}$, then $\left\|\nabla u^{m}\right\|_{2}^{2} \geq\left\|u^{m}\right\|_{\frac{m+p-1}{m}}^{\frac{m+p-1}{m}}$.

(iii) If $\left\|\nabla u^{m}\right\|_{2}^{2}<\left\|u^{m}\right\|_{\frac{m+p-1}{m}}^{\frac{m+p-1}{m}}$, then $u \in \stackrel{\overrightarrow{\mathcal{B}}}{\text {. }}$.

(iv) If $\left\|\nabla u^{m}\right\|_{2}^{2} \leq\left\|u^{m}\right\|_{\frac{m+p-1}{m}}^{\frac{m+p-1}{m}}$ and $\left\|\nabla u^{m}\right\|_{2} \neq 0$, then $u \in \mathcal{B} \cup \partial \mathcal{B}$.

Proof (i) Since $u \in \mathcal{S}$, we get from (1.9) and (1.10)

$$
\left\|\nabla u^{m}\right\|_{2}<\left(\frac{2 m(m+p-1)}{p-1-m} d\right)^{\frac{1}{2}}=C^{\frac{-(m+p-1)}{p-1-m}} \leq\left(\frac{\left\|u^{m}\right\|_{\frac{m+p-1}{m}}}{\left\|\nabla u^{m}\right\|_{2}}\right)^{\frac{-(m+p-1)}{p-1-m}}
$$

which implies $\left\|\nabla u^{m}\right\|_{2}>\left\|u^{m}\right\|_{\frac{m+p-1}{m}}^{\frac{m+p-1}{m}}$.

(ii) From $u \in \partial \mathcal{S}$ we get

$$
\left\|\nabla u^{m}\right\|_{2}=\left(\frac{2 m(m+p-1)}{p-1-m} d\right)^{\frac{1}{2}} \neq 0 .
$$

Then in the same way as the proof of (i), $\left\|\nabla u^{m}\right\|_{2}^{2} \geq\left\|u^{m}\right\|_{\frac{m+p-1}{m}}^{\frac{m+p-1}{m}}$ holds.

(iii) By (1.10) and $\left\|\nabla u^{m}\right\|_{2}^{2}<\left\|u^{m}\right\|_{\frac{m+p-1}{m}}^{\frac{m+p-1}{m}}$, we have

$$
\left\|\nabla u^{m}\right\|_{2}^{2}<\left\|u^{m}\right\|_{\frac{m+p-1}{m}}^{\frac{m+p-1}{m}} \leq C^{\frac{m+p-1}{m}}\left\|\nabla u^{m}\right\|_{2}^{\frac{m+p-1}{m}}
$$

which is equivalent to $\left\|\nabla u^{m}\right\|_{2}>C^{\frac{-(m+p-1)}{p-1-m}}$. So $u \in \mathcal{B}$.

(iv) In the same way as the proof of (iii), we have

$$
\left\|\nabla u^{m}\right\|_{2} \geq C^{\frac{-(m+p-1)}{p-1-m}}
$$

which implies $u \in \mathcal{B} \cup \partial \mathcal{B}$.

Lemma 2.3 Let $u$ be a solution of (1.5). Then the functional $E(u(t))$ defined as (1.6) is non-increasing in $t$. Moreover,

$$
\frac{4}{(m+1)^{2}} \int_{0}^{t}\left\||x|^{-\frac{s}{2}}\left(u^{\frac{m+1}{2}}(x, \tau)\right)_{\tau}\right\|_{2}^{2} d \tau+E(u(t))=E\left(u_{0}\right)
$$

Proof Multiplying the first equation of (1.5) with $\frac{1}{m}\left(u^{m}\right)_{t}$ and integrating over $\Omega \times(0, t)$, we get (2.2) and then that $E(u(t))$ is non-increasing in $t$ follows.

Lemma 2.4 Let $u$ be the solution of (1.5) with initial value $u_{0}$ such that $u_{0}^{m} \in H_{0}^{1}(\Omega)$ and $E\left(u_{0}\right) \leq d$. Then 
(i) $\left\|\nabla u^{m}\right\|_{2}^{2}>\left\|u^{m}\right\|_{\frac{m+p-1}{m}}^{\frac{m+p-1}{m}}$ if and only if $0<\left\|\nabla u^{m}\right\|_{2}<\left(\frac{2 m(m+p-1)}{p-1-m} d\right)^{\frac{1}{2}}$;

(ii) $\left\|\nabla u^{m}\right\|_{2}^{2}<\left\|u^{m}\right\|_{\frac{m+p-1}{m}}^{\frac{m+p-1}{m}}$ if and only if $\left\|\nabla u^{m}\right\|_{2}>\left(\frac{2 m(m+p-1)}{p-1-m} d\right)^{\frac{1}{2}}$.

Proof By (1.6), (2.2) and $E\left(u_{0}\right) \leq d$ we have

$$
\begin{aligned}
E(u(t)) & =\frac{p-1-m}{2 m(m+p-1)}\left\|\nabla u^{m}\right\|_{2}^{2}+\frac{1}{m+p-1}\left(\left\|\nabla u^{m}\right\|_{2}^{2}-\left\|u^{m}\right\|_{\frac{m+p-1}{m}}^{\frac{m+p-1}{m}}\right) \\
& \leq E\left(u_{0}\right) \leq d
\end{aligned}
$$

Then we can easily get (i) and (ii) from Lemma 2.2 and (2.3).

Lemma 2.5 Let $u$ be the solution of (1.5) with initial value $u_{0}$ such that $u_{0}^{m} \in H_{0}^{1}(\Omega)$ and $E\left(u_{0}\right) \leq d$. Then:

(i) $u(t) \in \mathcal{S}$ for $t \in[0, T)$ if $u_{0} \in \mathcal{S}$;

(ii) $u(t) \in \mathcal{B}$ for $t \in[0, T)$ if $u_{0} \in \mathcal{B}$;

where $\mathcal{S}$ and $\mathcal{B}$ are the sets defined in (1.12).

Proof (i) If the conclusion (i) is false, there must exist a time $t_{0} \in(0, T)$ such that $u\left(t_{0}\right) \in \partial \mathcal{S}$ and $u(t) \in \mathcal{S}$ for $0 \leq t<t_{0}$. Hence

$$
\left\|\nabla u^{m}\left(t_{0}\right)\right\|_{2}=\left(\frac{2 m(m+p-1)}{p-1-m} d\right)^{\frac{1}{2}}
$$

and

$$
\left\|\nabla u^{m}(t)\right\|_{2}<\left(\frac{2 m(m+p-1)}{p-1-m} d\right)^{\frac{1}{2}}, \quad t \in\left[0, t_{0}\right) .
$$

From (1.6), the second conclusion of Lemma 2.2 and (2.4), we obtain

$$
\begin{aligned}
E\left(u\left(t_{0}\right)\right) & =\frac{p-1-m}{2 m(m+p-1)}\left\|\nabla u^{m}\left(t_{0}\right)\right\|_{2}^{2}+\frac{1}{m+p-1}\left(\left\|\nabla u^{m}\left(t_{0}\right)\right\|_{2}^{2}-\left\|u^{m}\left(t_{0}\right)\right\|_{\frac{m+p-1}{m}}^{\frac{m+p-1}{m}}\right) \\
& \geq \frac{p-1-m}{2 m(m+p-1)}\left\|\nabla u^{m}\left(t_{0}\right)\right\|_{2}^{2}=d .
\end{aligned}
$$

By (2.4) and (2.5) we know that $\int_{0}^{t_{0}}\left\||x|^{-\frac{s}{2}}\left(u^{\frac{m+1}{2}}\right)_{t}\right\|_{2}^{2} d t>0$. Then it follows from (2.2) and (2.6) that $E\left(u_{0}\right)>E\left(u\left(t_{0}\right)\right) \geq d$, which contradicts $E\left(u_{0}\right) \leq d$.

(ii) The conclusion can be proved in the same way as (i).

Based on above preparations, we are ready to prove Theorem 1.2.

Proof of Theorem 1.2 (global existence part) We see from $E\left(u_{0}\right)=d$ and (1.6) that $\left\|\nabla u_{0}^{m}\right\|_{2}>$ 0 , which combines with $u_{0} \in \mathcal{S}$ and the first conclusion of Lemma 2.2 implies

$$
\left\|\nabla u_{0}^{m}\right\|_{2}^{2}>\left\|u_{0}^{m}\right\| \frac{\frac{m+p-1}{m}}{\frac{m+p-1}{m}} .
$$


Let $\lambda_{n}=1-\frac{1}{n}$ and $u_{0 n}=\lambda_{n} u_{0}$ for $n=2,3, \ldots$. Then it follows from (2.7), $\lambda_{n}<1$, and $m-p+1<0$ that

$$
\begin{aligned}
\left\|\nabla u_{0 n}^{m}\right\|_{2}^{2} & =\lambda_{n}^{2 m}\left\|\nabla u_{0}^{m}\right\|_{2}^{2}>\lambda_{n}^{2 m}\left\|u_{0}^{m}\right\|_{\frac{m+p-1}{m}}^{\frac{m+p-1}{m+1}}=\lambda_{n}^{m-p+1}\left\|u_{0 n}^{m}\right\|_{\frac{m+p-1}{m}}^{\frac{m+p-1}{m}} \\
& >\left\|u_{0 n}^{m}\right\|_{\frac{m+p-p-1}{m}}^{\frac{m+p-1}{m}}, \quad n=2,3, \ldots, \\
E\left(u_{0 n}\right) & =\frac{p-1-m}{2 m(m+p-1)}\left\|\nabla u_{0 n}^{m}\right\|_{2}^{2}+\frac{1}{m+p-1}\left(\left\|\nabla u_{0 n}^{m}\right\|_{2}^{2}-\left\|u_{0 n}^{m}\right\|_{\frac{m+p-1}{m}}^{\frac{m+p-1}{m}}\right) \\
> & 0, \quad n=2,3, \ldots
\end{aligned}
$$

Furthermore, by Lemma 2.1, there exists an integer $n_{*}$ such that $E\left(\lambda_{n} u_{0}\right)$ is strictly increasing for $n \leq n_{*}$, which means

$$
E\left(u_{0 n}\right)=E\left(\lambda_{n} u_{0}\right)<\lim _{n \rightarrow+\infty} E\left(\lambda_{n} u_{0}\right)=E\left(u_{0}\right)=d, \quad n=n_{*}, n_{*}+1, \ldots
$$

Equations (2.8)-(2.10) imply $u_{0 n} \in \Sigma_{1}$, where $\Sigma_{1}$ is defined as (1.11). Let $u_{n}$ be the solution of (1.5) with initial value $u_{0 n}$, then Theorem 1.1 implies $u_{n}$ exists globally such that

$$
u_{n}^{m}(t) \in L^{\infty}\left(0,+\infty ; H_{0}^{1}(\Omega)\right), \quad n=n_{*}, n_{*}+1, \ldots
$$

Similar to (2.3), for $0 \leq t<+\infty, n=n_{*}, n_{*}+1, \ldots$, we get

$$
\begin{aligned}
d> & E\left(u_{0 n}\right)=\frac{4}{(m+1)^{2}} \int_{0}^{t}\left\||x|^{-\frac{s}{2}}\left(u_{n}^{\frac{m+1}{2}}(x, \tau)\right)_{\tau}\right\|_{2}^{2} d \tau+E\left(u_{n}(t)\right) \\
= & \frac{4}{(m+1)^{2}} \int_{0}^{t}\left\||x|^{-\frac{s}{2}}\left(u_{n}^{\frac{m+1}{2}}(x, \tau)\right)_{\tau}\right\|_{2}^{2} d \tau \\
& +\frac{p-1-m}{2 m(m+p-1)}\left\|\nabla u_{n}^{m}\right\|_{2}^{2}+\frac{1}{m+p-1}\left(\left\|\nabla u_{n}^{m}\right\|_{2}^{2}-\left\|u_{n}^{m}\right\|_{\frac{m+p-1}{m}}^{\frac{m+p-1}{m}}\right) .
\end{aligned}
$$

Next, we will prove $\left\|\nabla u_{n}^{m}(t)\right\|_{2}^{2}>\left\|u_{n}^{m}(t)\right\|_{\frac{m+p-1}{m}}^{\frac{m+p-1}{m}}$ for $0 \leq t<+\infty$. If not, it follows from (2.8) that there exists $t_{*}>0$ such that $\left\|\nabla u_{n}^{m}\left(t_{*}\right)\right\|_{2}^{2}=\left\|u_{n}^{m}\left(t_{*}\right)\right\|_{\frac{m+p-p}{m}}^{\frac{m+p-1}{m}}$. Then it follows from (1.9) that $E\left(u_{n}\left(t_{*}\right)\right) \geq d$, which contradicts $E\left(u_{n}\left(t_{*}\right)\right)<d$ by (2.12). Then from (2.12), we obtain

$$
\begin{aligned}
& \int_{0}^{t}\left\||x|^{-\frac{s}{2}}\left(u_{n}^{\frac{m+1}{2}}(x, \tau)\right)_{\tau}\right\|_{2}^{2} d \tau<\frac{d(m+1)^{2}}{4}, \\
& 0 \leq t<+\infty, n=n_{*}, n_{*}+1, \ldots, \\
& \left\|u_{n}^{m}(t)\right\|_{\frac{m+p-1}{m+p-1}}^{\frac{m}{m}} \leq\left\|\nabla u_{n}^{m}(t)\right\|_{2}^{2} \leq \frac{2 m(m+p-1)}{p-1-m} d, \\
& 0 \leq t<+\infty, n=n_{*}, n_{*}+1, \ldots
\end{aligned}
$$

From (2.13), (2.14), and the compactness method in [9], it follows that there exist $u$ and a subsequence $\left\{u_{k}\right\}$ of $\left\{u_{n}\right\}$ such that for all $T>0$
1. $u \in L^{\infty}\left(0, T ; H_{0}^{1}(\Omega)\right)$ and $\int_{0}^{T}\left\||x|^{-\frac{s}{2}}\left(u^{\frac{m+1}{2}}(x, t)\right)_{t}\right\|_{2}^{2} d t \leq \frac{d(m+1)^{2}}{4}$,
2. $u_{k} \rightarrow u$ a.e. on $\Omega \times(0, T)$, 
3. $u_{k}^{m} \rightarrow u^{m}$ weakly star in $L^{\infty}\left(0, T ; H_{0}^{1}(\Omega)\right)$,

4. $u_{k} \rightarrow u$ weakly star in $L^{\infty}\left(0, T ; L^{m+p-1}(\Omega)\right)$,

5. $|x|^{-\frac{s}{2}}\left(u_{k}^{\frac{1+m}{2}}\right)_{t} \rightarrow|x|^{-\frac{s}{2}}\left(u^{\frac{1+m}{2}}\right)_{t}$ weakly in $L^{2}\left(0, T ; L^{2}(\Omega)\right)$.

Then it follows from the construction of $u_{n}$ that $u$ is a global solution of (1.5) and $u(t) \in \overline{\mathcal{S}}$ for $0 \leq t<\infty$.

Proof of Theorem 1.2 (blow-up part) Let $u(t)$ be the solution of problem (1.5) with initial value $u_{0}$ satisfying $E\left(u_{0}\right)=d$ and $u_{0} \in \mathcal{B}$. We need to show that the maximal existence time $T$ of $u$ is finite. We assume $T=+\infty$ and prove the conclusion by contradiction. Let

$$
f(t)=\frac{1}{m+1} \int_{0}^{t} \int_{\Omega}|x|^{-s}|u(x, \tau)|^{m+1} d x d \tau
$$

Then

$$
f^{\prime \prime}(t)=\int_{\Omega}|x|^{-s} u^{m} u_{t} d x=-\left\|\nabla u^{m}\right\|_{2}^{2}+\left\|u^{m}\right\|_{\frac{m+p-1}{m}}^{\frac{m+p-1}{m+}}
$$

From (2.2), (2.15), and

$$
E(u(t))=\frac{p-1-m}{2 m(m+p-1)}\left\|\nabla u^{m}(t)\right\|_{2}^{2}+\frac{1}{m+p-1}\left(\left\|\nabla u^{m}(t)\right\|_{2}^{2}-\left\|u^{m}(t)\right\|_{\frac{m+p-1}{m}}^{\frac{m+p-1}{m}}\right)
$$

we get

$$
\begin{aligned}
f^{\prime \prime}(t)= & \frac{p-1-m}{2 m}\left\|\nabla u^{m}\right\|_{2}^{2}-(m+p-1) E\left(u_{0}\right) \\
& +\frac{4(m+p-1)}{(m+1)^{2}} \int_{0}^{t}\left\||x|^{-\frac{s}{2}}\left(u^{\frac{m+1}{2}}(x, \tau)\right)_{\tau}\right\|_{2}^{2} d \tau .
\end{aligned}
$$

By $u_{0} \in \mathcal{B}$ and Lemma (2.5), we obtain $u(t) \in \mathcal{B}$ for $0 \leq t<+\infty$, i.e.,

$$
\left\|\nabla u^{m}(t)\right\|_{2}>\left(\frac{2 m(m+p-1)}{p-1-m} d\right)^{\frac{1}{2}}, \quad 0 \leq t<+\infty .
$$

From (2.17), (2.18) and $E\left(u_{0}\right)=d$ we obtain $f^{\prime \prime}(t)>\frac{4(m+p-1)}{(m+1)^{2}} \int_{0}^{t}\left\||x|^{-\frac{s}{2}}\left(u^{\frac{m+1}{2}}(x, \tau)\right)_{\tau}\right\|_{2}^{2} d \tau$. The remaining part of the proof is the same as that in [5].

\section{Conclusion}

In this paper, we study a singular porous medium equation considered in [5], where the global existence and blow-up conditions were got for the case of subcritical initial energy $E\left(u_{0}\right)<d$. We complete the results by studying the global existence and blow-up conditions for the case of critical initial energy $E\left(u_{0}\right)=d$. 


\section{Acknowledgements}

This work is partially supported by the Fundamental Research Funds for the Central Universities grant XDJK2015A16, NSFC grant 11201380, Project funded by China Postdoctoral Science Foundation grant 2014 M550453 and the Second Foundation for Young Teachers in Universities of Chongqing.

Received: 11 January 2016 Accepted: 8 April 2016 Published online: 13 April 2016

\section{References}

1. Vazquez, JL: The Porous Medium Equation. Oxford Mathematical Monographs. Clarendon, Oxford (2010)

2. Wu, Z, Zhao, J, Yin, J, Li, H: Nonlinear diffusion equations. World Scientific, River Edge, NJ (2001)

3. Zhong, T: Non-Newton filtration equation with special medium void. Acta Math. Sci. 24B(1), 118-128 (2004)

4. Wang, Y: The existence of global solution and the blowup problem for some $p$-Laplace equations. Acta Math. Sci. 27(2), 274-282 (2007)

5. Zhou, J: A multi-dimension blow-up problem to a porous medium diffusion equation with special medium void. Appl. Math. Lett. 30, 6-11 (2014)

6. Zhou, J, Yang, D: Upper bound estimate for the blow-up time of an evolution $m$-Laplace equation involving variable source and positive initial energy. Comput. Math. Appl. 69(2), 1463-1469 (2015)

7. Zhou, S, Zheng, S: Global blow-up in a degenerate and strongly coupled parabolic system with localized sources. Comput. Math. Appl. 60(9), 2564-2571 (2010)

8. Hu, Y, Li, J, Wang, L: Blow-up phenomena porous medium equation with nonlinear flux on the boundary. J. Appl. Math. 2013(8), 1-5 (2013)

9. Lions, JL: Quelques methodes de resolution des problemes aux limites non lineaires. Dunod, Paris (1969)

\section{Submit your manuscript to a SpringerOpen ${ }^{\odot}$ journal and benefit from:}

- Convenient online submission

- Rigorous peer review

Immediate publication on acceptance

- Open access: articles freely available online

- High visibility within the field

- Retaining the copyright to your article 